\title{
A Novel Approach for Finger Vein Pattern Enhancement using Gabor and Canny Edge Detector
}

\author{
Kayode A. Akintoye \\ Image Processing Researcher \\ Department of Computer \\ Science, Universiti Teknologi \\ Malaysia (UTM).81310 Skudai \\ Johor, Malaysia
}

\author{
M. Rahim M. Shafry \\ Professor of Image \\ Processing/ Multimedia Data \\ Management \\ Department of Computer \\ Science, Universiti Teknologi \\ Malaysia (UTM).81310 Skudai \\ Johor, Malaysia
}

\author{
Abdul Hanan Abdullah \\ Professor of Network Security \\ Senior Members of IEEE / \\ ACM \\ Department of Computer \\ Science, Universiti Teknologi \\ Malaysia (UTM). 81310 Skudai \\ Johor, Malaysia
}

\begin{abstract}
Biometrics is the science of identifying a person using physiological or behavioural features. A biometric verification system authenticates an individual's identity by matching the live biometric template to his/her biometric template or templates stored in the system database. Nevertheless, finger vein verification system is cost effective in comparison but high in accuracy with opportunities of fake detection and biocryptography system. Previous research on finger vein verification has proved that it's accuracy relies on enhancement of vein image pattern quality. However, at times noise generates as a result of hair and skin texture, which is as well enhanced because they are much resembles the vein structure. Noise is any process that affects the original image since it is not part of the original image. This causes great error when extracting accurate vein patterns. It also leads to increasing processing time during finger vein extraction, which is eventually cause an inaccuracy in matching/verification system. To overcome this problem, a novel approach for finger vein pattern enhancement using Gabor-Canny edge detector is proposed, which is far better and more accurate than previous method.
\end{abstract}

\section{Keywords}

Noise, Finger vein detection, ROI, Gabor filter, Canny edge detector

\section{INTRODUCTION}

In current age of Information security technology, there is widespread uses of biometric technologies such as fingerprint, veins, face, voice, and face recognition in various access control, automatic teller machines (ATMs), financial transactions and forensics [1]. Biometric finger vein verification has been proved to be the best as it can solve many shortcomings of other biometric technologies, such drawbacks like fingerprint sweat effect, scars and distortions; face recognition illumination and pose change. Thou, finger vein biometric system is a little costly in comparison with other biometric systems but it is high in accuracy with opportunities of fake detection and bio-cryptography system [2]. Finger vein has patterns inside human fingers that makes person's identity unique. The vein imaging system depends on the uses of near-infrared (NIR) light illumination at a wavelength of $750 \mathrm{~mm}$ or more, because veins absorb deoxyhemoglobin at this range $[3,4]$. Previous research works has shown that the accuracy of finger vein biometric verification and recognition system lies on enhancement of quality image vein pattern and extraction of accurate various features in order to use finger vein as a factor for personal recognition system in multimodal system, as well as detecting fake finger veins.

Image information is the main target for image processing. An Image consists of different information of a scene such as the size, colour, and orientation of different objects presents in that scene. Image processing shows a significant part in finger vein verification operation. It is the planning of an image for features extraction and later for analysis use. Finger vein images are not always clear when captured due to number of factors. It can sometimes show some irregularity shadings and highly saturated regions, thus caused noise. A noise can be defined as a process that affects the original image since it is not a part of the original image [5]. This can cause great error when extracting accurate vein patterns. It can also lead to increasing processing time during finger vein extraction, which is eventually cause an inaccuracy of matching or verification system. Thus, it is necessary to apply noise removal algorithm to enhance the quality of the degraded image. The noise removal algorithms reduce or remove the visibility of noise by smoothing the entire image leaving areas near contrast boundaries [5].

The noise can be as a result of any the following factors:

- Blurry image, which is patterns of vein that contain little contrast;

- Askew image, which is vein images with definite grade of deformation;

- Dim image, which is dim or black portion in the images;

- Bright image, which is sunny portion in the images.

Recently, research has shown that noise can occur as a result of non-uniform illumination, low local contrast, hairs and skin texture [6]. However, at time noise generates as a result of hair and texture, which are as well enhanced because it is much resembles the vein structure.

Biometric verification system consists of four various phases, which are: data collection, preprocessing, feature extraction and matching/verification. The process of the preprocessing module comprises the normalization of the image, detection of Region of Interest (ROI), and enhancement of vein pattern, which lead to image segmentation. The image pre-processing stages is shown in figure 1 . In section 2 of this paper, the related work and explanation of ROI were introduced. Section 3 contains vein enhancement using edge detector and its result, while section 4 gives conclusion. 


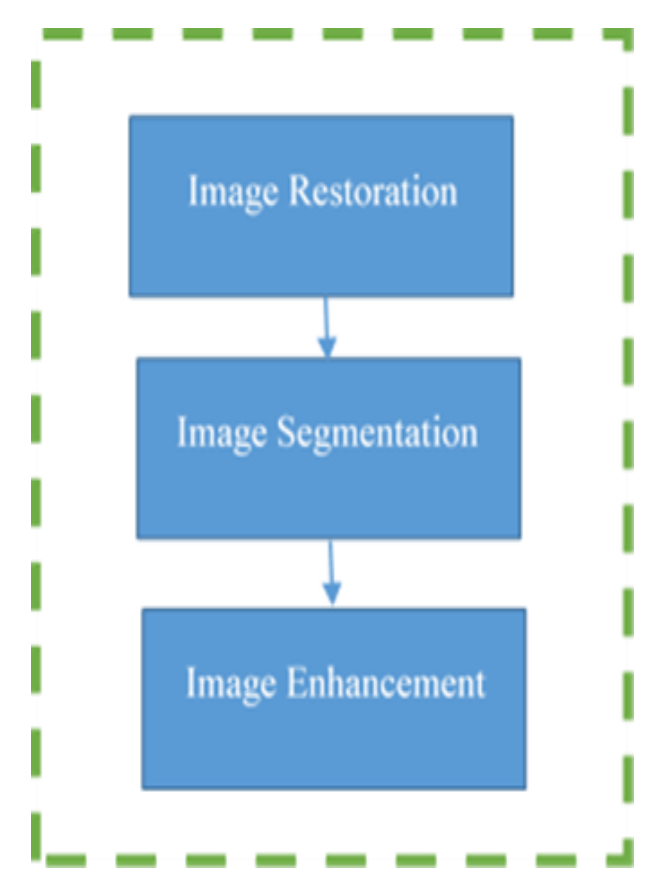

Fig 1: Image Preprocessing Stages

\section{FINGER VEIN RELATED WORKS AND ITS MODEL}

This section explains the existing research works on finger vein recognition and its models.

\subsection{Related Works}

A number of approaches which implement this common architecture have already been offered starting from the revolutionary work of Park et al. [7]. In Im et al work [8], the conventional vein pattern recognition algorithm contains of a preprocessing part, which applying sequentially an iterative Gaussian low pass, a high pass, and a modified median filter, a recognition part which includes the extraction of the binary veins via local thresholding, and finally the matching between the individual patterns.

An improved vein pattern extraction algorithm was proposed in [9]. The algorithm balances the loss of vein patterns in the edge area, gives more enhanced and stabilized vein pattern information. The result shows better performance than the existing algorithms. Furthermore, the difficult emerging from the iterative nature of filtering preprocess is solved by designing a filter that is processed only once, increasing significantly the recognition speed and reducing the hardware complexity. The proposed algorithm is implemented with an FPGA device and the false acceptance rate is five times better than the existing algorithm and the recognition speed is measured to be 100 (ms/person). In [10] and [11], finger vein images were enhanced by utilizing restoration algorithms which reduces the scattering effect created through IR image acquisition. Extraction of region of interest (ROI) is expel from the original images in a definite proportion to work with. This extraction has some noteworthy benefits. It helps as a pre-processing to take out the interpretation and resolution of vein images presented in the data collection process. It likewise takes out the most informative region in the images. Thou, it cuts a great deal of data amount however without losing much valuable information. This speeds up the feature extraction and matching or verification procedure.
Kumar and Zhou [12] pre-processed the finger vein images obtained from the totally contactless imaging by normalized it. They systematically developed an original method for the finger-vein feature extraction using Gabor filters. Miural et all in 2011[13] used repeated line racking to tract the local dark position of image but it was difficult to define a shape for variable width veins. While Cho et al in 2011[14] used an adaptive version of Gabor filter to enhance the distinctiveness region in the original finger vein image. Yang et al [10] produced enhanced finger vein image by restoration-based method through consideration $\mathrm{n}$ effect of the layered structured of skin. They finally restored the images using a point-spread function (PSF) and a biological optical model (BOM) (15). Park et al [16] proposed an image enhancement method using an optimal Gabor filter based on the directions and thickness of the vein line. However, the width of veins and hair that resembles vein were not considered. In modification, Shin et al in 2014 [4], proposed in using Gabor filter in combination with Retinex filter, which is fuzzy-based fusion. Gupta \& Gupta [6], reduced the effect of noise occurred due to non-uniform illumination and low local contrast using Multiscale Matched filtering but this method refused to deal with the noise generated by hair and texture, which are closely resembles the vein structure.

\subsection{Finger Vein Model}

Finger vein technology mechanisms by distinguishing the vein designs in a person's finger. The person's finger is place over a scanner and the near-infrared light maps the area of the veins. The red cells present in the veins integrate the rays and appear on the guide as dark lines, while the remaining finger structure looks as white. This vein example is then checked against a prerequisite example to confirm the person. As veins are inside in the body and have an abundance of separating elements, endeavors to fashion a character are amazingly troublesome, in this approach of empowering an abnormal state of security. Figure 1 establishes the general procedures of the ID model utilizing finger veins biometrics. After image capture, a miniature range of a finger image is situated at the region of interest (ROI) to separate the components and to analyze distinctive fingers. Utilizing the segments inside ROI for verification can enhance the computation proficiency altogether.

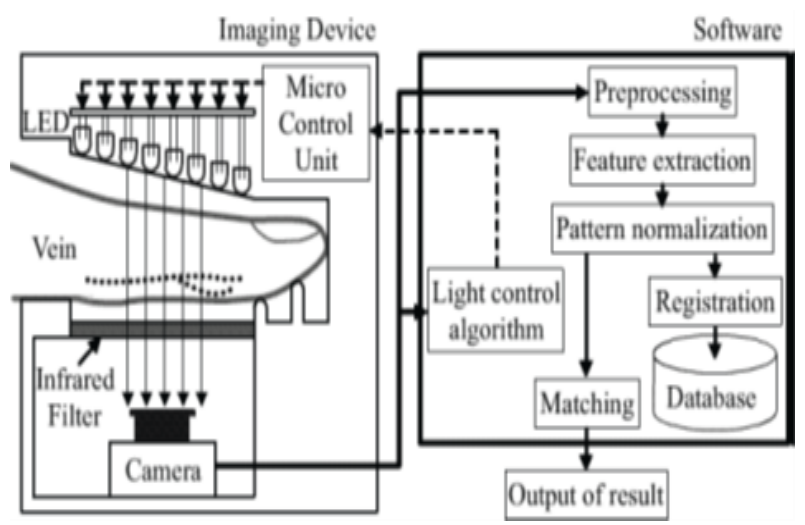

(a) FV Verification System Architecture [17] 


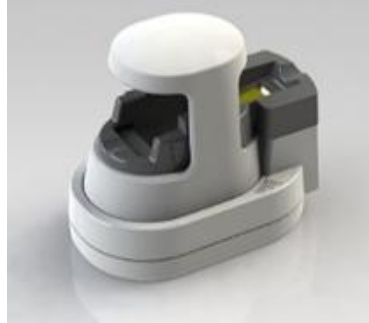

(b) Finger-vein imaging device

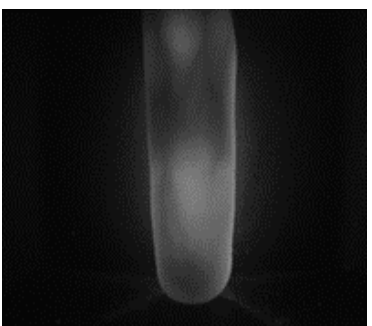

(c) Example of infrared image of finger-vein

Fig 2: The finger-vein based verification system [17]

\section{VEIN ENHANCEMENT AND PROPOSED METHOD}

As indicated in Gupta [6], enhanced vein image was utilized for vein matching straightforwardly without experience vein extraction. The enhanced vein image was encoded by utilizing radon transform and matched with the neural network system. Additionally, in [17], improved vein image was facilitated by using organize just relationship. Furthermore, surface (by using compcode) of the enhanced vein image was used for highlighting encoding as a piece of [18]. Algorithms which does not use vein extraction gives exact results if finger veins are acquired under controlled environment. In any case, these can be impacted by the issues of commotion, non-uniform illumination, low neighborhood differentiation and position assortments.

\subsection{Proposed Methodology}

Finger vein image, the sample, which contains in figure 4a of a subject from Universiti Sains Malaysia (FV-USM) image database [18] was used under Gabor filter [19] and Canny edge detector [20] for extraction of edges the pattern vein. FV-USM finger vein dataset is a public dataset, which was developed by light transmission method, the finger is placed in between the infra-red (IR) source and image sensor. From two periods, 5904 total images from 492 finger classes were obtained. The captured finger images have spatial and depth resolution of $640 \times 480$ and 256 grey levels, separately [23].

The Gabor filter is defined as:

$\mathrm{g}(\chi, \mathrm{y}, \lambda, \theta, \varphi, \sigma, \gamma)=\exp \left(-\frac{\chi^{\prime^{2}}+\gamma^{2}+\mathrm{y}^{\prime^{2}}}{2 \sigma^{2}}\right) \cos \left(2 \Pi \frac{\chi^{\prime}}{\lambda}+\varphi\right)$

Where,

$\mathrm{x}^{\prime}=\mathrm{x} \cos \theta+\mathrm{y} \sin \theta$

$\mathrm{y}^{\prime}=-\mathrm{x} \sin \theta+\mathrm{y} \cos \theta$

The " $\lambda$ " represent wavelength of the sinusoidal factor, " $\theta$ " represents the orientation, and " $\psi$ " is the phase offset, " $\sigma$ " is the standard deviation, " $\gamma$ " is the spatial ratio.

Edge is considered to be one of the best important features of images in the computer vision. Edge can create a border round the objects by protecting the information connected to the shapes of them which can easily differentiate between the object and its background [21]. Thus, edge detection techniques play an important role in the analysis of quality and modality of images. From the literature survey we can observe that the "Canny Edge Detection Algorithm" is the optimal algorithm among the edge detection algorithms. The three main criteria of the canny edge detection are as follows:
From the literature. we observe that the "Canny Edge Detection Algorithm" is the ideal algorithm among the edge detection algorithms. The three fundamental criteria of the canny edge detection are as per the following [22]:

1. Low error rate: This means that edge that occurs in image must not be neglected, so also, non-edge should not be attended to.

2. Good Localization: Here, the actual edge distance and that of edge pixels' distance found by the detector must be minimized.

3. Single Response: This is a case whereby single edge has one response.

The Canny Edge Detection algorithm mainly has five steps in it. They are:

Step i: Computing the horizontal (Gx) and vertical (Gy) gradient of each pixel in an image.

Step ii: Using the above information the magnitude $(\mathrm{G})$ and direction (of each pixel in the image is calculated.

Step iii: In this step all non-maxima's are made as zero that is suppression the non-maxima's thus the step is called Non-Maximal Suppression. Step iv: The high and low thresholds are measured using the histogram of the gradient magnitude of the image.

Step v: To get the proper edge map hysteresis thresholding is employed which will link between the weak and strong edges. The weak edges are taken into consideration if and only if it is connected to one of the strong edges or else it is eliminated from the edge map. The strong edge is the one whose pixel is greater than the high threshold and weak edge is one whose pixel value lays between high and low threshold.

The proposed algorithm is summarized as follows:

i. Read an image captured under near infrared light illumination finger vein image from the image database.

ii. Get the Pruned border of input image and store it

iii. Get the boundaries of input image and store it.

iv. Get the region of interest of input image by normalization and store it.

v. Now apply the Gabor filter with passed and accepted parameter. Perform Canny edge detector on the image as a resultant of the Gabor filter.

vi. These operations are useful to extract edges of the pattern vein 


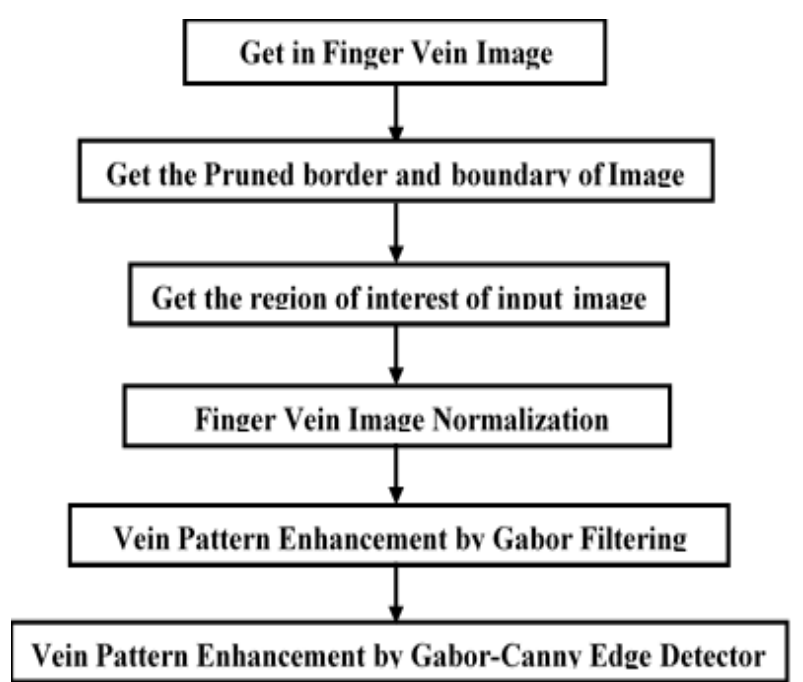

Fig 3: The proposed model of Finger vein pattern extraction using Gabor-Canny Filter

\subsection{Result and Discussion}

The experimentation focused on enhancement of the vein from the raw finger vein image (figure 4a), which leads to extraction of finger vein features for a robust verification system. The figure $4 \mathrm{~b}$ shows the finger vein image pruned border while figure $4 \mathrm{c}$ displays the finger vein dilated boundary. The dilated boundary was cropped by normalization and gives region of interest (ROI) of finger vein as displays in figure $4 \mathrm{~d}$. The Gabor and Canny edge detector has been used in association to extract the vein features from edges of the vein line lies at the finger region of the finger as shown in figure $4 \mathrm{e}$ and $4 \mathrm{f}$.

This paper has aimed at extract the edges of the finger vein pattern out of original image. The Gabor filter was used to extract the interest area from the original image. The mathematical form as shown in equation (1) of Gabor filter was exercised by the set of parameter such as " $\lambda$ " represent wavelength of the sinusoidal factor, " $\theta$ " represents the orientation, and " $\psi$ " is the phase offset, " $\sigma$ " is the standard deviation, " $\gamma$ " is the spatial ratio. On passing certain threshold values to this parameter will extract the interest set out of original image.

The quality of a finger vein image usually lies on the quantity and the contrast of the vein patterns. The figure $4 \mathrm{f}$ demonstrates the case of high quality finger vein image, which contain more vein patterns examples with higher contrast. These vein patterns can be roughly regarded as a mix of an arrangement of line segments, which are identified by Canny edge identifier. The results summaries are shown as follows:
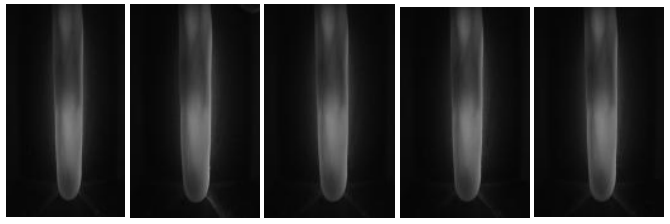

(a)Original Finger Vein Image

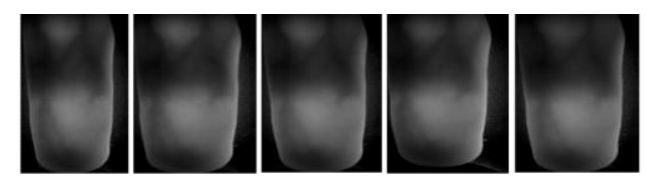

(b) Finger Vein Image Pruned border

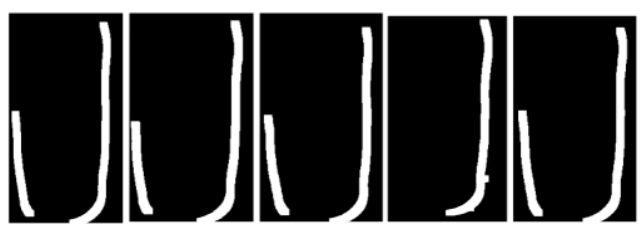

(c) Finger vein Dilated boundary
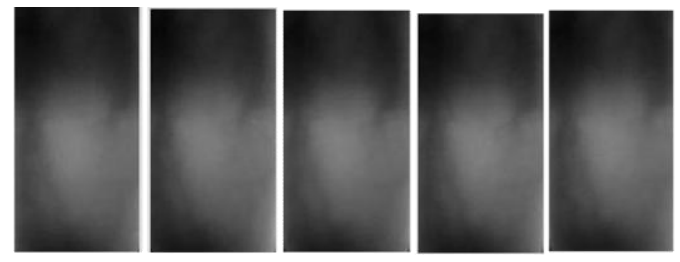

(d) Finger Vein Image Region of Interest (ROI) Extraction by Cropped Normalized
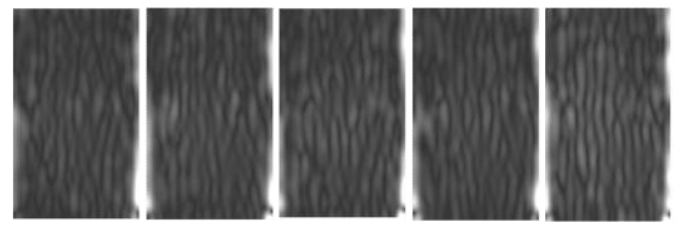

(e) Finger vein pattern enhancement using Gabor filter
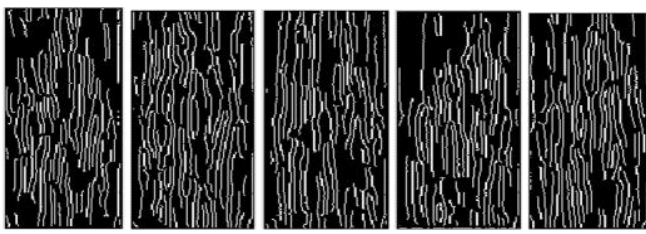

(f) Finger vein pattern enhancement using Gabor-Canny edge detector

Fig 4(a-f): Segmentation stages of Finger Vein preprocessing

\section{CONCLUSION}

The experimental results presented in section 3 consistently indicate that the proposed method of finger vein pattern enhancement, using Gabor-Canny edge detector, achieves significantly improved pattern image, which can further use for better features extraction at minimal processing time. The use of Gabor filter and Canny edge detector prominently lead to highlight and visualize as well as to extract the vein pattern edges out of original image.

In paper, the causes of noise in finger vein image are stated. Also, it has fully been established that thou Gabor filter can used to enhance finger vein images pattern but using Canny edge detector in addition to it yielded better result in processing noise created by skin hair, which much seem like vein. This method is the systematical development of a new approach for the finger vein pattern enhancement using Gabor-Canny edge detector. 


\section{REFERENCES}

[1] T. D. Pham, Y. H. Park, D. T. Nguyen, S. Y. Kwon, K. R. Park, "Nonintrusive Finger-Vein Recognition System Using NIR Image Sensor and Accuracy Analyses According to Various Factors", Sensors, 15(7):16866-94, 2015.

[2] W. Yang, J. Hu, S. Wang, "A finger-vein based cancellable bio-cryptosystem", Network and System Security: Springer, p. 784-90, 2013.

[3] J. Yang, J. Yang, editors. "Multi-channel gabor filter design for finger-vein image enhancement. 2009 Fifth International Conference on Image and Graphics, IEEE, 2009.

[4] K. Y. Shin, Y. H. Park, D. T. Nguyen, K. R. Park, "Finger-vein image enhancement using a fuzzy-based fusion method with gabor and retinex filtering", Sensors, 14(2):3095-129, 2014

[5] A. A. A. Hamid, R. Kumoi, M. S. M. Rahim, N. Z. Syazrah, "Fingerprint Image Enhancement Using Median Sigmoid Filter", Jurnal Teknologi, 75(4), 2015.

[6] P. Gupta and P. Gupta, "An accurate finger vein based verification system", Digital Signal Processing, 38:43-52, 2015.

[7] G. Park, S. Im, H. Choi, editors. "A person identification algorithm utilizing hand vein pattern", Proc of Korea Signal Processing Conf; 1997.

[8] S. K. Im, H. M. Park, Y. W. Kim, S. C. Han, S. W. Kim, C. H. Kang, et al. "An biometric identification system by extracting hand vein patterns", JOURNAL-KOREAN PHYSICAL SOCIETY, 38(3):268-72, 2001.

[9] S. K. Im, H. M. Park, S. W. Kim, C. K. Chung, H. S. Choi, editors, "Improved vein pattern extracting algorithm and its implementation", Consumer Electronics, 2000 ICCE 2000 Digest of Technical Papers International Conference on, IEEE, 2000.

[10] J. Yang, Y. Shi, "Finger-vein ROI localization and vein ridge enhancement", Pattern Recognition Letters, 33(12):1569-79, 2012.

[11] J. Yang, Y. Shi, "Towards finger-vein image restoration and enhancement for finger-vein recognition", Information Sciences, 268:33-52, 2014.

[12] A. Kumar, Y. Zhou, "Human identification using finger images", Image Processing, IEEE Transactions on, 21(4):2228-44, 2012.
[13] N. Miura, A. Nagasaka, T. Miyatake, "Feature extraction of finger-vein patterns based on repeated line tracking and its application to personal identification", Machine Vision and Applications, 15(4):194-203, 2004

[14] S. R. Cho, Y. H. Park, G. P. Nam, K. Y. Shin, H. C. Lee, K. R. Park, et al., editors, "Enhancement of finger-vein image by vein line tracking and adaptive gabor filtering for finger-vein recognition" Applied Mechanics and Materials, Trans Tech Publ., 2011.

[15] J. Yang, B. Zhang, Y. Shi, "Scattering removal for finger-vein image restoration", Sensors, 12(3):3627-40, 2012.

[16] Y. H. Park, K. R. Park, "Image quality enhancement using the direction and thickness of vein lines for fingervein recognition", International Journal of Advanced Robotic Systems, 9, 2012

[17] B. Huang, Y. Dai, R. Li, D. Tang, W. Li, editors, "Finger-vein authentication based on wide line detector and pattern normalization", Pattern Recognition (ICPR), 20th International Conference on, IEEE, ; 2010.

[18] M. S. M. Asaari, S. A. Suandi, B. A. Rosdi, "Fusion of band limited phase only correlation and width centroid contour distance for finger based biometrics", Expert Systems with Applications, 41(7):3367-82, 2014.

[19] A. M. Al-juboori, W. Bu, X. Wu, Q. Zhao, "Palm vein verification using gabor filter" International Journal of Computer science issues, 10(1):678-84, 2013.

[20] S. Raut, V. Humbe, "An Approach to Boundary Extraction of Palm Lines and Vein Pattern", International Journal of Image, Graphics and Signal Processing (IJIGSP), 6(12):47, 2014.

[21] A. Sharifara, M. Rahim M. Shafry, H. Sayyadi, "A Robust Human Face Detection Algorithm Based on Skin Color Segmentation and Edge Detection", Journal of Theoretical \& Applied Information Technology, 77(1), 2015.

[22] A. L. KABADE, "Canny edge detection algorithm", International Journal of Advanced Research in Electronics and Communication Engineering (IJARECE), 5(5):1292-1295, 2016.

[23] FV-USM Finger Vein Image Database. Available online: http://blog.eng.usm.my/fendi/?page_id=262 